\title{
Analysis and Research on the Starting Process of Control Algorithm for Certain Type of Aviation Auxiliary Power Unit
}

\author{
Ya-nan Guo ${ }^{1,2}$, Ding Fan ${ }^{1,2}$, Kai Peng ${ }^{1,2}$, \\ ${ }^{1}$ School of Power and Energy, Northwestern \\ Polytechnical University, \\ Xi'an Shaanxi, China \\ ${ }^{2}$ Advanced Aircraft Engine Collaborative Innovation \\ Center, \\ Beijing, China
}

\author{
Qiu-xia Wang ${ }^{3}$ \\ ${ }^{3}$ Avic Aero-engine Control Research Institute, \\ Wuxi Jiangsu, China
}

\begin{abstract}
The control system of a certain type APU is analyzed and researched. Certain analysis on the mechanism of the structure and working principle of APU and study of the idea of its fuel control system are made. The main fuel flow control law and control algorithm are deduced by reverse analysis method. The formation principle is analyzed and the physical meaning is uncovered of interpolation table which involved in the control algorithm. The design principle of main fuel control law and control algorithm for this type of auxiliary power unit is mastered. It's a valuable reference for the future design of the control system of both aero-engine and auxiliary power unit.
\end{abstract}

Keywords-auxiliary power unit; starting process; control algorithm; interpolation table fitting.

\section{INTRODUCTION}

Auxiliary power unit (APU) is a set of independent small gas turbine power plant which is installed on the aircraft. It is not depend on any energy from outside the machine, and mainly used for output electric power, shaft power and pressurized gas source, in order to meet modern aircraft the engine start, the air emergency, the cabin air conditioner and other auxiliary energy supply requirements. APU is an important and indispensable part of today's aircraft systems. Based on a certain type of single rotor full authority digital electronic control system of auxiliary power unit, this type of APU's structure and working principle are analyzed; The main fuel flow control law and control algorithm are studied; finally least square method is used to fit several key interpolation table's function and parameter in the control algorithm, the high fitting precision is obtained and its physical significance is revealed; this type of APU's control law and relevant design idea of its algorithm are studied. It is of great significance that reduces not only the design and research cycle of aircraft engine and APU control system but also the risk and cost of its design process.

\section{ANALYSIS ON APU CONTROL SYSTEM}

\section{A. APU structure and working principle}

The APU belongs to single rotor structure, mainly including two basic parts which is APU core engine and transmission gear box. For the structure, the core engine is a single-stage centrifugal plus vane diffuser compressor, reflux annular combustor and a single-stage radial turbine. The compressor and turbine are connected coaxial, while the exhaust pipe which is of diffusion structure has a muffler to reduce the noise of the high-speed gas. Transmission gearbox will reduce the speed of high-speed turbine, and connect the starter, fuel systems, oil system, generator and cooling fan together. APU operating mode includes start mode, available mode, main engine start mode, loop control mode and stop mode.

\section{B. APU control system structure}

This type of APU control system architecture is shown in Fig.1. It is mainly composed of digital electronic controller, gas turbine power plant, fuel supply device, starter generator, hydraulic load device, monitor computer, sensors and so on. All parts contact each other and work together.

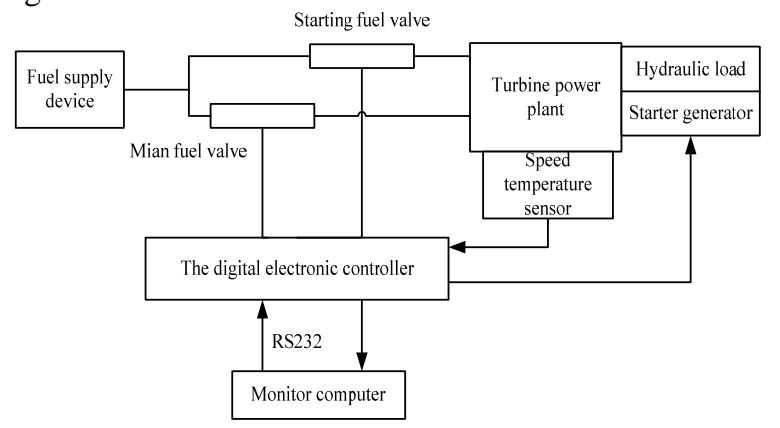

Fig.1. APU control system structure

\section{THE STARTING PROCESS OF THE MAIN FUEL CONTROL LAW AND CONTROL ALGORITHM}

The type of the APU in starting process is using time sequence regularity to control the APU, just as shown in Fig.2. Controller first according to the size of the current APU rotor rotational speed calculates the corresponding expectation value of exhaust temperature, and subtracts the expected exhaust gas temperature and the current exhaust temperature. Then the contoller according to the current APU rotating speed and the fuel electric current which compute from the inlet condition calculate the upper and lower limits, then calculated according to the current APU rotating speed and inlet condition fuel 
electric current of the upper and lower limits, and then through the variable parameter PI algorithm to calculate the fuel torque motor current value, and then get the current time needed by the APU fuel flow. The control law is: $W_{f}=f\left(T_{5}, n\right)$, and the exhaust gas temperature $T_{5}$ is the controlled variable, the fuel flow $W_{f}$ is control variable.

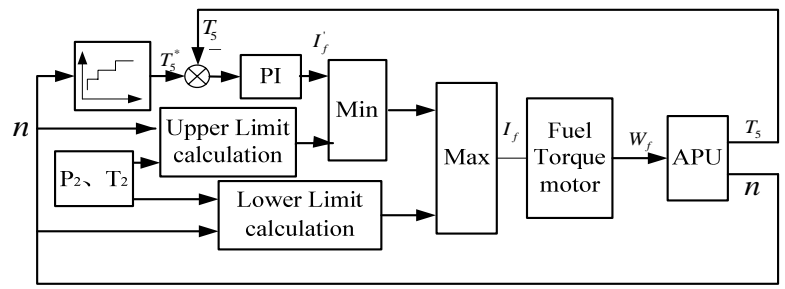

Fig.2. The main fuel flow control law principle block diagram of starting process

The main fuel flow of starting process is calculated as shown in formula 1. Among them, $f_{1}(x), f_{2}(x), f_{3}(x)$ respectively instead of interpolation table 1,2,3, Similarly, $f_{i}(x)$ represent interpolation table $\mathrm{i}$ in the remaining part. The calculation process of program is that if it appears the situation that the compressor inlet pressure $P_{2}$ crossborder, the interpolation table 1 is used; if the exhaust temperature $T_{5}$ is more than $703{ }^{\circ} \mathrm{C}$, the interpolation table 2 is used; if each input parameter is not out of bounds, the interpolation table 3 is used to calculate the expected exhaust gas temperature according to the current speed. Known from the analysis of calculation, process of fuel flow calculation adopts the variable parameter PI controller and proportional integral coefficients $K_{P}, K_{I}$ are changed with APU compressor inlet air pressure $P_{2}$ and temperature changes of $T_{2}$, and the specific proportion integral coefficient calculation process is shown in formula 2. $f_{4}(x), f_{5}(x)$ respectively express interpolation table 4 and interpolation table 5. $P_{2} L S B$ is the compressor inlet pressure correction coefficient and its specific numerical are known by program. When $P_{2}$ crosses border, $P_{2}=6.76$ Psia. $T_{2}$ LSB is the compressor inlet temperature correction coefficient, and its specific numerical is known by program, when $T_{2}$ crosses border, $T_{2}=6.7^{\circ} \mathrm{C} . k, k_{1}, k_{2}, k_{3}$ are constant, and these are all known by the data program.

$$
\left\{\begin{array}{l}
\Delta w f=K_{P} \Delta e_{T 5}(k)+K_{I} e_{T 5}(k) \\
w f=\Delta w f+w f \_l a s t \\
T_{5}^{*}(k)=f_{1 o r 2 o r 3}[N(k)] \\
e_{T 5}(k)=T_{5}^{*}(k)-T_{5}(k) \\
\Delta e_{T 5}(k)=e_{T 5}(k)-e_{T 5}(k-1)
\end{array}\right.
$$

$$
\left\{\begin{array}{c}
K_{P}=\frac{f_{4}\left(P_{2} \_ \text {corr }\right)}{T_{2-c o r r}} \times k_{p} \\
K_{\mathrm{I}}=\frac{f_{5}\left(P_{2} \text { corr }\right)}{T_{2} \text { corr }} \times k_{i} \\
P_{2-c o r r}=\frac{P_{2}}{P_{2} L S B} \times k_{1} \\
T_{2-c o r r ~}=\left(\frac{T_{2}}{T_{2} L S B}+k_{2}\right) \times k_{3}
\end{array}\right.
$$

Combining with the Simulink simulation model and the embedded S function, the starting process calculation of upper limit fuel is shown in formula 3 , and the calculation of lower limit is shown in formula 4. $\varepsilon_{\min }$ is the compressor inlet pressure correct fuction, $\theta_{\min }$ is the compressor inlet temperature corrected fuction. In the formula 3, if $P_{2}$ beyonds the normal range of specified in the program, $P_{2}=3.4 \mathrm{Psia}$; if $T_{2}$ beyonds the normal range of specified in the program, $T_{2}=54.1^{\circ} \mathrm{C}$; if $P_{2}$ crosses the bound, the interpolation table 6 is used; if $P_{2}$ does not cross the bound, the interpolation table 7 is used. In the formula 4, if $P_{2}$ crosses the bound, $P_{2}=14.7$ Psia ; if $T_{2}$ crosses the bound, $T_{2}=63.7^{\circ} \mathrm{C}$;if $P_{2}$ crosses the bound, the interpolation table 8 is use; if $P_{2}$ does not crosses the bound, the interpolation table 9 is used.

$$
\begin{aligned}
& \left\{\begin{array}{c}
\text { Wf_upper }=\frac{f_{12}\left(\varepsilon_{\min }\right) \times f_{6 \circ 7}(n)}{\theta_{\min } \times 4} \times k \\
\varepsilon_{\min }=f_{13}\left(P_{2}\right) \times k_{1} \\
\theta_{\min }=\left(\frac{T_{2}}{T_{2} L S B}+k_{2}\right) \times k_{3}
\end{array}\right. \\
& \left\{\begin{array}{c}
\text { Wf_lower }=\frac{f_{12}\left(\varepsilon_{\max }\right) \times f_{80 r 9}(N)}{\theta_{\max } \times 8} \times k \\
\varepsilon_{\text {max }}=f_{13}\left(P_{2}\right) \times k_{1} \\
\theta_{\max }=\left(\frac{T_{2}}{T_{2} L S B}+k_{2}\right) \times k_{3}
\end{array}\right.
\end{aligned}
$$

\section{FORMATION PRINCIPLE OF THE INTERPOLATION TABLE 12 AND TABLE 13}

Through the analysis and research, preliminary presumed parameter is designed as followed:

Selectting the specific working points from the flight envelope. The specific working points are determined by inlet temperature and inlet pressure, there is a one-to-one correspondence between height and mach number. The control parameter for the starting process is adjusted in the vicinity of each working point according to certain control performance index, such as dynamic and static error and overshoot, and then the fitting of the control parameter is carried out at all working points. if the 
scheduling rule of it and known control parameter are consistent, preliminary conclusion about the adjustment of the parameter control law is same as the original design.

There are several ways for fitting or interpolation for gain scheduling control parameters, and fitting based on the exponential function is a common way among them. Therefore the exponential function is used as fitting function of curve interpolation table. Firstly, hypothesis function curve is a regular form of exponential function as shown in formula 5 .

$$
y=a \times(x / b)^{c}
$$

According to the physical meaning of abscissa and ordinate of interpolation table 12, the correction parameters $\quad\left(P_{s d} / P_{2} L S B\right) \times k_{1}$ and normal atmospheric pressure ${ }^{P_{s d}}$ substitute the value of $a$ and ${ }^{b}$ respectively, then the data is used to optimize c by the least square method. Hereby the expression of $f_{12}\left(\varepsilon_{\min }\right)$ is confirmed as shown in formula 6 .

$$
f_{12}\left(\varepsilon_{\min }\right)=\left(\frac{f_{13}\left(P_{2}\right) \times k_{1}}{\frac{P_{s t d}}{P_{2} \_S B} \times k_{1}}\right)^{0.5} \times \frac{P_{s t d}}{P_{2}-L S B} \times k_{1}
$$

The same type of formula 5 is adopt Interpolation table 13, and then the selection of $a$ and $b$ values is made according to the abscissa and ordinate's physical meaning, and using the least square method the data is used to optimize and determine the value of $c$ finally, the resultant expression of $f_{13}\left(P_{2}\right)$ is shown in formula 7.

$$
f_{13}\left(P_{2}\right)=\left(\frac{P_{2}}{P_{s t d}}\right)^{0.5849} \times \frac{P_{s t d}}{P_{2} \_S B}
$$

Physical meaning of the interpolation table 12 and interpolation table 13 are the correction of the compressor inlet pressure $P_{2}$. The fitted curve and the original curve as shown in Fig. 3 and Fig.4, and the error between them is neglectable, which demonstrates preliminary hypothesis.

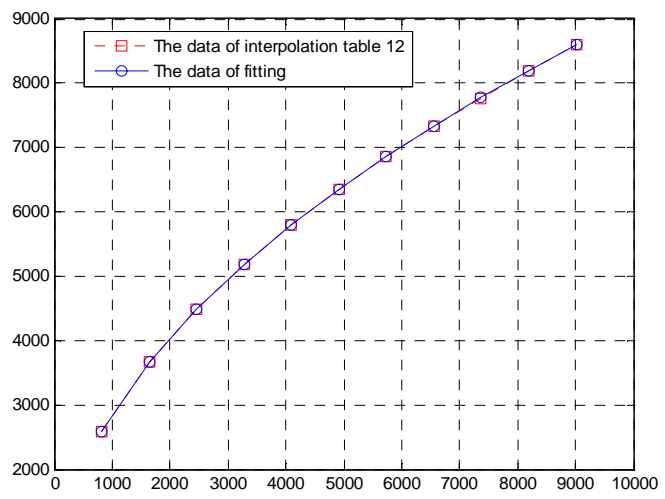

Fig.3. Fitting curve of interpolation table 12

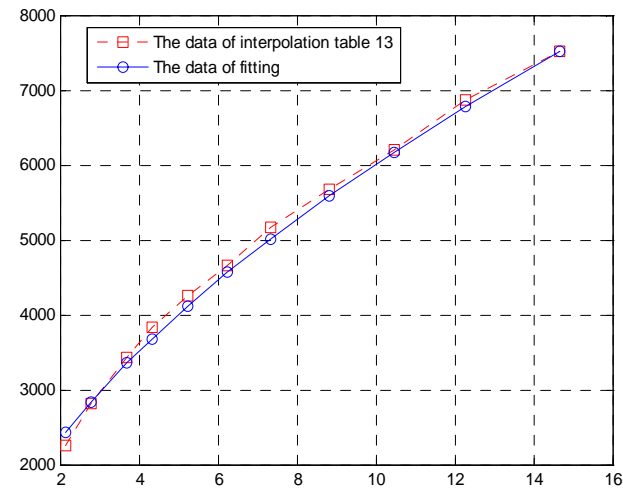

Fig.4. Fitting curve of interpolation table 13

\section{CONCLUSIONS}

The full authority digital electronic control system of a certain single rotor auxiliary power unit is taken as a research object, control law and the control algorithm of the main fuel flow were derived by reverse analysis combined with the working principle and the recorded data of working process of the APU. The results show that: The precision of the interpolation table deduced is higher, the reducibility of physical meanings is good, and analysis and study of control law and control algorithm are feasible. Analysis results can provide valuable reference for the future design of the control system of engine and auxiliary power unit.

\section{REFERENCES}

[1] Li Dongjie. Application Status and Development Trend of Auxiliary Power Unit:Aviation Science and Technology, 2012.

[2] Fan Siqi. Aero engine control [M]. Xi 'an: Northwestern Polytechnical University Press, 2008.

[3] Yao Hua. Full authority digital engine control system [M]. Beijing: Aviation Industry Press, 2013.

[4] Zhou Wenxiang. Aircraft engine and control system modeling and object oriented simulation study [D]. PhD thesis of Nanjing University of Aeronautics and Astronautics, 2006. 